\title{
Deoxyribozyme-nanosponges for improved photothermal therapy by overcoming thermoresistance
}

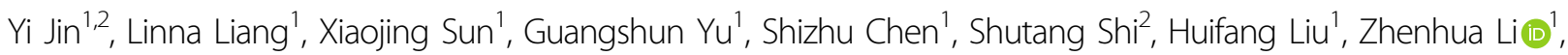 \\ Kun Ge', Dandan Liu', Xinjian Yang ${ }^{1}$ and Jinchao Zhang ${ }^{1}$
}

\begin{abstract}
Thermoresistance shields cancer cells from damage by hyperthermia, decreasing the efficacy of photothermal therapy. Therefore, the design of photothermal therapeutic systems that avoid inducing thermoresistance is highly desirable for precision medicine. Herein, by simply assembling a cationic polymer and a long single strand of DNA encoded with multivalent deoxyribozyme (or DNAzyme) sequences, we have developed a sponge-like nanoplatform for highly efficient photothermal therapy that uses DNAzymes to overcome thermal resistance. The multivalent customized DNAzymes can catalytically cleave HSP70 mRNAs and downregulate the expression of their proteins, which protect MCF-7 cells from damage by hyperthermia. In vitro and in vivo studies have demonstrated the capability of this therapeutic platform to sensitize MCF-7 cells to heat by inhibiting the overexpression of HSP70. In addition, the nanoplatform can act as an efficient multimode imaging agent to trace its accumulation in tumor tissue.
\end{abstract}

\section{Introduction}

Acquired or intrinsic cancer therapeutic resistance has been one of the major challenges in clinical practice. The mechanisms involved in cancer therapeutic resistance protect cancer cells from being killed by chemodrugs or other agents, resulting in a limited therapeutic effect ${ }^{1-3}$. Therefore, the need for efficient cancer therapy has fueled the development of innovative approaches to preventing therapeutic resistance. Advances in nanotechnology have made nanomaterials promising candidates for the development of therapeutic agents ${ }^{4-7}$. Appropriately sized nanomaterials could be endowed with the ability to achieve many objectives that are difficult to address with chemodrugs. In particular, nanoparticles with near-

\footnotetext{
Correspondence: Xinjian Yang (jianxinyang123@163.com) or Jinchao Zhang (jczhang6970@163.com)

${ }^{1}$ College of Chemistry and Environmental Science, Chemical Biology Key Laboratory of Hebei Province, Key Laboratory of Medicinal Chemistry and Molecular Diagnosis of the Ministry of Education, Hebei University, No. 180 Wusidong Road, 071002 Baoding, China

${ }^{2}$ College of Medical Science, Hebei University, No. 180 Wusidong Road, 071002 Baoding, China
}

infrared (NIR) light-to-heat conversion capabilities have attracted interest for use in photothermal cancer therapy. To date, a variety of nanomaterials including noble metalbased nanomaterials, carbon-based nanomaterials, and copper sulfide nanoparticles have been widely employed as photothermal therapy (PTT) agents ${ }^{8-14}$. As a promising alternative or supplement to traditional cancer therapy, PTT is minimally invasive and compensates for the reduced therapeutic efficacy caused by chemodrug resistance. In addition, PTT can be combined with other therapeutic methodologies, such as chemotherapy and gene therapy, to achieve high therapeutic efficacy against cancer ${ }^{15-17}$. Nevertheless, recent studies have indicated that PTT is always accompanied by a heat shock response that shields cancer cells from damage by hyperthermia, decreasing its therapeutic efficacy ${ }^{18-20}$. To enhance therapeutic efficacy, a higher irradiation power or larger administration dose is required, which may result in severe side effects due to the overheating of neighboring normal tissue. Therefore, the ability to kill cancer cells

\section{(c) The Author(s) 2018}

(c) (i) Open Access This article is licensed under a Creative Commons Attribution 4.0 International License, which permits use, sharing, adaptation, distribution and reproduction cc) in any medium or format, as long as you give appropriate credit to the original author(s) and the source, provide a link to the Creative Commons license, and indicate if changes were made. The images or other third party material in this article are included in the article's Creative Commons license, unless indicated otherwise in a credit line to the material. If material is not included in the article's Creative Commons license and your intended use is not permitted by statutory regulation or exceeds the permitted use, you will need to obtain permission directly from the copyright holder. To view a copy of this license, visit http://creativecommons.org/licenses/by/4.0/. 
without inducing thermoresistance by PTT remains challenging.

The heat shock protein (HSP) family has been indicated as a central factor that contributes to thermoresistance during cancer PTT by inhibiting hyperthermia-induced apoptosis ${ }^{21,22}$. During the heat shock response, the expression levels of most HSPs are increased, leading to a decrease in the efficacy of PTT. Attempts have been made to induce dysfunction of HSPs by introducing small molecular inhibitors ${ }^{18,23}$. Although these attempts were promising in anticancer therapy, severe side effects may occur due to their intrinsic toxicity. An alternative approach for improving photothermal efficiency involves inhibiting the expression of HSPs by interfering with their genes $^{24,25}$. DNAzymes are more attractive than other gene therapeutic agents because of their easy, low-cost synthesis, high catalytic efficiency and selectivity. DNAzymes can be designed to bind to a complementary target mRNA and cleave $\mathrm{it}^{26}$. Herein, we have developed a DNAzymenanosponge therapeutic platform to enable the highly efficient photothermal treatment of cancer by catalytically

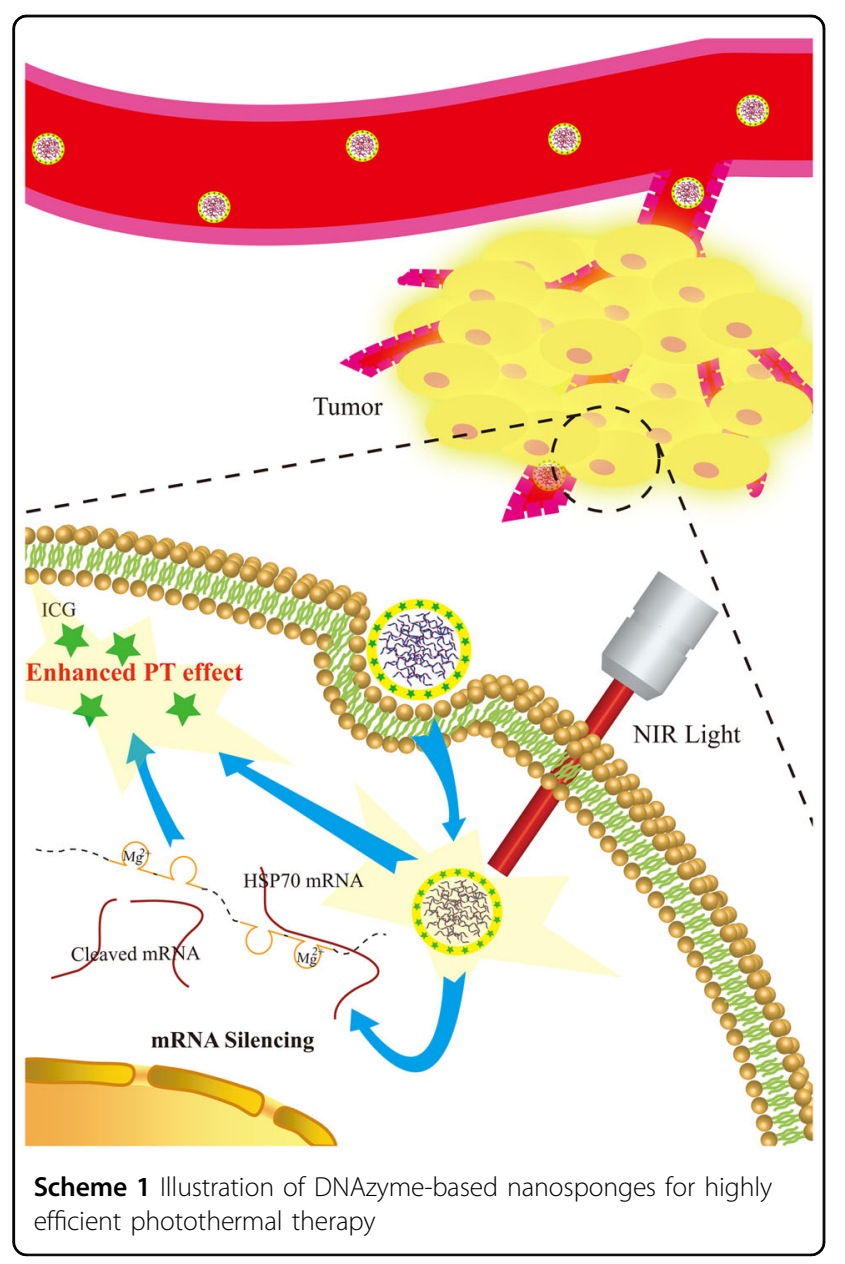

restraining the expression of HSPs. As shown in Scheme 1, the photothermal small molecule indocyanine green (ICG), an FDA-approved NIR cyanine dye, was used for $\mathrm{PTT}^{27}$. The nanotherapeutic platform, which could absorb ICG in a sponge-like manner, was constructed easily by assembling a cation polymer and long singlestranded DNA with repeated DNAzyme domains. The nanosponges were of an appropriate size for accumulation at the tumor via an enhanced permeability and retention (EPR) effect. The multivalence of the DNAzymes that were incorporated into the nanosponge allowed the effective attenuation of the thermoresistance by silencing the HSP gene. In addition, the intrinsic biodegradability of this nanotherapeutic platform would eliminate concerns regarding their potential long-term toxicity.

\section{Materials and methods}

Rolling cycle amplification (RCA) of HSP70 DNAzyme

All HPLC-purified oligonucleotides used in this work (Table S1) were synthesized by Sangon Biotech Co., Ltd. (Shanghai, China). The long single-stranded HSP70 DNAzyme was synthesized by RCA as described in our previous work $^{26}$. Fluorescein-12-dUTP $(0.1 \mathrm{mM})$ was also added for synthesize the fluorescein isothiocyanate (FITC)-labeled HSP70 DNAzymes (FITC-Dz). The HSP70 DNAzymes were precipitated by ethyl alcohol and sodium acetate.

\section{In vitro cleavage activity of nanosponges}

Nanosponges were dissolved to a concentration of $5 \mu \mathrm{M}$ in $50 \mu \mathrm{L}$ of simulated lysosomal fluid buffer $(142 \mathrm{mg} / \mathrm{L}$ $\mathrm{Na}_{2} \mathrm{HPO}_{4}, 6.65 \mathrm{~g} / \mathrm{L} \quad \mathrm{NaCl}, 62 \mathrm{mg} / \mathrm{L} \quad \mathrm{Na}_{2} \mathrm{SO}_{4}, 29 \mathrm{mg} / \mathrm{L}$ $\mathrm{CaCl}_{2} \cdot \mathrm{H}_{2} \mathrm{O}, 250 \mathrm{mg} / \mathrm{L}$ glycine, $8.09 \mathrm{~g} / \mathrm{L}$ potassium phthalate, $\mathrm{pH} 4.5$ ) and sonicated for $15 \mathrm{~s}$. Following incubation at $37^{\circ} \mathrm{C}$ for $0,30,60,90,120,150,180,210$, and $240 \mathrm{~min}$, the $\mathrm{pH}$ of the nanosponge suspensions was adjusted to neutral by adding $750 \mu \mathrm{L}$ of PBS (pH 7.4). The catalytic activity of the nanosponge suspensions was detected by the hydrolysis rate of a diribonucleotide within a fluorescent nucleic acid probe. The concentration of the probe mRNA was $2.5 \mu \mathrm{M}$. The fluorescence intensity was measured using a fluorescence spectrophotometer (Hitachi, F7000, Japan).

\section{Synthesis and characterization of nanosponge-ICG nanoparticles}

The synthesis of nanosponge-ICG nanoparticles was carried out via the following steps. First, $2.5 \mu \mathrm{M}$ HSP70 DNAzyme was mixed with branched PEI (25k) at N/P ratios of 5,10 , and 20 for $1 \mathrm{~h}$ in aqueous solution. Excess PEI was removed by centrifugation $(5000 \mathrm{rpm}, 5 \mathrm{~min})$ to obtain PEI-coated DNAzyme (nanosponge). Then, after centrifugation, the nanosponge was further loaded with ICG (Macklin, Shanghai, China) using a similar method. 
For this purpose, $0.2 \mathrm{mg} / \mathrm{mL}$ ICG in aqueous solution was mixed with the nanosponge precipitate under vigorous stirring. The final nanoparticles (nanosponge-ICG) were obtained by centrifugation $(10,000 \mathrm{rpm}, 10 \mathrm{~min})$ and resuspended in ultrapure water.

The loading efficiency (LE) of ICG in the nanosponge was detected as follows. Fresh nanosponge was isolated from aqueous suspension by ultracentrifugation $(10,000$ rpm, $10 \mathrm{~min}$ ). Non-loaded ICG in the suspending liquid was removed. Then, the concentration of ICG loaded in the nanosponge was determined at the wavelength of 775 $\mathrm{nm}$ by a microplate reader. The LE was calculated by the following formula: $\mathrm{LE}$ of ICG $=\left[\mathrm{ICG}_{(\text {Total })}-\mathrm{ICG}_{\text {(Non-loa- }}\right.$ ded) $] / I_{C G_{(\text {Total }}} \times 100$.

The morphologies of nanosponge-ICG were determined by scanning electron microscopy (SEM, JEOL, JSM7500F, Japan) and transmission electron microscopy (TEM, FEI, Tecnai G2, USA). Energy dispersive spectra (EDS) was used to analyze the composition of the nanosponge-ICG. The zeta potential was determined using a Nano-ZS (Malvern Instruments) system with disposable cuvettes. The UV/Vis absorption spectra of free ICG and nanosponge-ICG were obtained using a UV/ Vis spectrophotometer (U2900, Hitachi, Japan).

\section{In vitro photothermal efficiency}

One milliliter of nanosponge-ICG solution (containing $0.05,0.1,0.2 \mathrm{mg} / \mathrm{mL}$ ICG) was added to a centrifuge tube. The samples were irradiated for $6 \mathrm{~min}$ with $0.2,0.4,0.8$, and $1.2 \mathrm{~W} / \mathrm{cm}^{2}$ NIR light, and the temperature changes were simultaneously recorded by an infrared thermal imaging camera.

\section{Hemolysis assay of nanosponge-ICG nanoparticles}

Blood was freshly drawn from a male ICR mouse by cardiac puncture. A $10 \% \mathrm{w} / \mathrm{v}$ red blood cell (RBC) solution was obtained. Then, the $0.1 \mathrm{~mL} \mathrm{RBC}$ suspension was mixed with $0.9 \mathrm{~mL}$ of nanosponge-ICG nanoparticle solution $(2.5-10 \mu \mathrm{M})$. After $30 \mathrm{~min}$ of incubation, the mixture was centrifuged $(3000 \mathrm{rpm}, 10 \mathrm{~min})$ to remove non-lysed RBCs. The absorbance of the released hemoglobin was determined at the wavelength of $570 \mathrm{~nm}$ by a microplate reader.

\section{Cell culture}

MCF-7 cells were purchased from the cell bank of Peking Union Medical College (Beijing, China), and incubated in Dulbecco's Modified Eagle's Medium (Life Technologies, Carlsbad, CA, USA) supplemented with $10 \%(\mathrm{v} / \mathrm{v})$ FBS, $100 \mathrm{U} / \mathrm{mL}$ penicillin, and $100 \mathrm{mg} / \mathrm{mL}$ streptomycin in a $37^{\circ} \mathrm{C}, 5 \% \mathrm{CO}_{2}$ atmosphere. For HSP detection, cellular uptake, PCR, and western blot analysis, cells $\left(10^{5}\right.$ cells per well) were precultured in 6-well plates for $24 \mathrm{~h}$ before use.

\section{Cellular uptake and subcellular localization of nanosponge-ICG}

After being seeded in 6-well culture plates, cells were incubated with FITC-Dz $(2.5 \mu \mathrm{M})$, ICG $(0.05 \mathrm{mg} / \mathrm{mL})$, or nanosponge-ICG $(2.5 \mu \mathrm{M}$, containing $0.05 \mathrm{mg} / \mathrm{mL}$ ICG) for 1, 2, 4, and $8 \mathrm{~h}$. The uptake of FITC-Dz, ICG, and nanosponge-ICG into the cells was analyzed by a FACScalibur flow cytometer.

Lyso-Tracker Red and Hoechst 33342 probe (Thermo Fisher Scientific, Waltham, MA, USA) were used to determine the subcellular localization. In brief, MCF-7 cells were treated with $2.5 \mu \mathrm{M}$ of nanosponge-ICG for $4 \mathrm{~h}$. Next, the cells were stained with Lyso-Tracker dyes. The subcellular localization was detected by confocal laser scanning microscopy (CLSM) (Olympus, IX81, Japan). The fluorescence was imaged at excitation wavelengths of $350 \mathrm{~nm}$ for Hoechst 33342, $577 \mathrm{~nm}$ for Lyso-Tracker Red, $488 \mathrm{~nm}$ for FITC, and $633 \mathrm{~nm}$ for ICG.

\section{Heat shock response detection}

Briefly, MCF-7 cells were treated with $0.05 \mathrm{mg} / \mathrm{mL}$ ICG and incubated for $8 \mathrm{~h}$. The cells were harvested at $0,1,2$, 4 , and $8 \mathrm{~h}$ after NIR light irradiation $\left(808 \mathrm{~nm}, 1 \mathrm{~W} / \mathrm{cm}^{2}, 5\right.$ min), and the HSP70 gene expression was determined by real-time PCR and western blot.

\section{Cell viability assay}

Cells were seeded $\left(1 \times 10^{5}\right.$ cells per well $)$ in a 96-well plate and incubated with $2.5 \mu \mathrm{M}$ DNAzyme, $0.05 \mathrm{mg} / \mathrm{mL}$ ICG, $2.5 \mu \mathrm{M}$ con-nanosponge-ICG (a nanosponge without HSP70 DNAzyme, containing $0.05 \mathrm{mg} / \mathrm{mL}$ ICG), or $2.5 \mu \mathrm{M}$ nanosponge-ICG (containing $0.05 \mathrm{mg} / \mathrm{mL}$ ICG) for $24 \mathrm{~h}$. After being washed three times, the cells were illuminated with NIR light $\left(808 \mathrm{~nm}, 1 \mathrm{~W} / \mathrm{cm}^{2}, 5 \mathrm{~min}\right)$. After further culture for $24 \mathrm{~h}$ further culture, an MTT assay was conducted at a wavelength of $570 \mathrm{~nm}$ using a microplate reader (Molecular Devices, VersaMax, USA).

\section{Real-time PCR analysis}

MCF-7 cells were incubated with $0.05 \mathrm{mg} / \mathrm{mL}$ ICG, 2.5 $\mu \mathrm{M}$ con-nanosponge-ICG, or $2.5 \mu \mathrm{M}$ nanosponge-ICG (both containing $0.05 \mathrm{mg} / \mathrm{mL}$ ICG) for $24 \mathrm{~h}$. After washing three times with PBS, the cells were immersed in fresh culture medium and illuminated with NIR light $(808 \mathrm{~nm}$, $\left.1 \mathrm{~W} / \mathrm{cm}^{2}, 5 \mathrm{~min}\right)$. After further treatment for $24 \mathrm{~h}$, total RNA was extracted by Trizol reagent (Invitrogen, Carlsbad, CA, USA). Quantitative PCR was performed using Power SYBR Green PCR Master Mix (Thermo Scientific, Waltham, MA, USA). The used primer DNA sequences are listed in Table S2.

\section{Western blot analysis}

After the same treatment described in the section on real-time PCR analysis, cells were lysed in buffer (100 
$\mathrm{mmol} / \mathrm{L}$ tris- $\mathrm{HCl} \mathrm{pH}$ 7.4, $1 \%$ TritonX-100, $150 \mathrm{mmol} / \mathrm{L}$ $\mathrm{NaCl}, 10 \mu \mathrm{g} / \mathrm{mL}$ aprotinin, $1 \mathrm{mM}$ phenylmethanesulfonylfluoride, $10 \mu \mathrm{g} / \mathrm{mL}$ leupeptin). Total cellular proteins were separated by SDS-PAGE and transferred to a polyvinylidene fluoride membrane, then blocked. After this step, polyclonal anti-HSP70 antibody (Proteintech, Wuhan, China) was added. The final detection was performed by ChemiDoc XRS+ with Image Lab software (Bio-RAD).

\section{Animal xenograft model}

All the experiments were conducted in accordance with the institutional animal use and care regulations. For inoculation, approximately $1 \times 10^{7}$ MCF-7 cells were subcutaneously injected into female BALB/c nude mice (Weitong Lihua, Beijing, China). ICG $(0.1 \mathrm{mg} / \mathrm{L})$, connanosponge-ICG, and nanosponge-ICG (with ICG content of $0.1 \mathrm{mg} / \mathrm{L}$ ) stock solutions were prepared for animal experiments.

\section{In vivo study of the biodistribution of ICG and nanosponge-ICG}

Three groups of mice were intravenously injected with $100 \mu \mathrm{L}$ of saline, ICG, or nanosponge-ICG for imaging. Then, the major organs were harvested for biodistribution analysis. Detection was conducted on a LI-COR Odyssey Infrared Imaging System (MSOT inVision 128, iThera Medical, Germany).

\section{In vivo PTT}

An in situ intratumoral experiment was conducted on ambilateral tumor-bearing mice by injecting $100 \mu \mathrm{L}$ of ICG or nanosponge-ICG. Then, the tumors were irradiated with NIR light $\left(808 \mathrm{~nm}, 2 \mathrm{~W} / \mathrm{cm}^{2}, 10 \mathrm{~min}\right)$ after $8 \mathrm{~h}$. The tumor temperature was recorded using an infrared thermal camera. To evaluate the in vivo antitumor effect, MCF-7 tumor-bearing mice (tumor volume approximately $100 \mathrm{~mm}^{3}$ ) were randomly divided into eight groups (six per group). They were injected with $100 \mu \mathrm{L}$ of saline, DNAzyme, ICG, ICG, con-nanosponge-ICG, connanosponge-ICG, nanosponge-ICG, or nanosponge-ICG through the tail vein. The mice were then irradiated with NIR light $\left(2 \mathrm{~W} / \mathrm{cm}^{2}, 10 \mathrm{~min}\right)$.

\section{Histological examination}

The major organs and tumor tissues were excised and processed into paraffin slices. Then, the sliced tissues were stained with hematoxylin and eosin (H\&E) for histological examination.

\section{Hematotoxicity}

Blood and tissues were harvested from mice intravenously injected with DNAzyme $(5 \mu \mathrm{M})$, ICG $(0.1 \mathrm{mg} / \mathrm{mL})$, $5 \mu \mathrm{M}$ con-nanosponge-ICG, and $5 \mu \mathrm{M}$ nanosponge-ICG (containing $0.1 \mathrm{mg} / \mathrm{mL}$ ICG) 15 days post injection. Eight important cardiac, hepatic, and kidney function indicators (LDH, HBDH, CK, ALT, AST, UA, BUN, Cr) were measured.

\section{Immunohistochemistry and TUNEL assay}

HSP70 expression was analyzed using immunohistochemistry. Tissue slices were incubated with rabbit polyclonal antibody anti-HSP70 at $4{ }^{\circ} \mathrm{C}$ overnight. Immunohistochemical staining of HSP70 was performed using a streptavidin-biotin-peroxidase complex and diaminobenzidine. The results were evaluated by the immunohistochemical score. Apoptosis in the tumor tissue was determined by the TUNEL assay.

\section{Results and discussion}

\section{Characterization of DNAzyme-nanosponge}

In the current study, the first step in the construction of DNAzyme-nanosponges involved the preparation of a long single strand of DNA with repeated DNAzyme domains using a method (Fig. S1) ${ }^{28}$. The RCA template was customized with two HSP70 DNAzymes that catalytically cleave the HSP70 gene at different sites for highly efficient gene silencing. Then, nanosponges with a size of approximately $68 \mathrm{~nm}$, as determined by DLS (Fig. S2c), were constructed by simply assembling the long single strand of DNA and branched PEI. The SEM and TEM images (Fig. 1a, S2) indicate that the nanosponges were monodisperse due to the amino groups on the surface. The EDS results show that these nanosponges are mainly composed of the elements $\mathrm{C}, \mathrm{P}, \mathrm{O}$, and N (Fig. S3). The zeta potential in Fig. 1b provides potent evidence for the successful synthesis of the nanosponge-ICG. Initially, the DNAzymes obtained by RCA were negatively charged. After incubation with PEI, the zeta potential increased from $-36 \mathrm{mV}$ to $19.7 \mathrm{mV}$. The positive charge of these nanosponges guaranteed their high adsorption of negatively charged small molecules. However, the LE of these nanosponges for the photothermal agent ICG reached a maximum at an N/P ratio of 10 . To perform highly efficient cancer therapy, nanosponges with an $\mathrm{N} / \mathrm{P}$ ratio of 10 were employed in the subsequent experiments. Because the typical absorption peak of ICG was located in the NIR region, the typical absorption peak of nanosponge-ICG remained in the NIR region (Fig. 1c). The photothermal conversion property of the nanosponge-ICG was determined by irradiation with $808 \mathrm{~nm}$ light. As shown in Fig. 1d, the temperature of the nanosponge-ICG increased by $28.1{ }^{\circ} \mathrm{C}$ after exposure to NIR light for $6 \mathrm{~min}$. However, the temperature of water increased by only $3.2^{\circ} \mathrm{C}$. Then, we tested the photothermal conversion efficiency by altering the concentration of the nanosponge-ICG as well as the irradiation energy. The temperature trends for this material are shown in Fig. S4 and demonstrate that the 

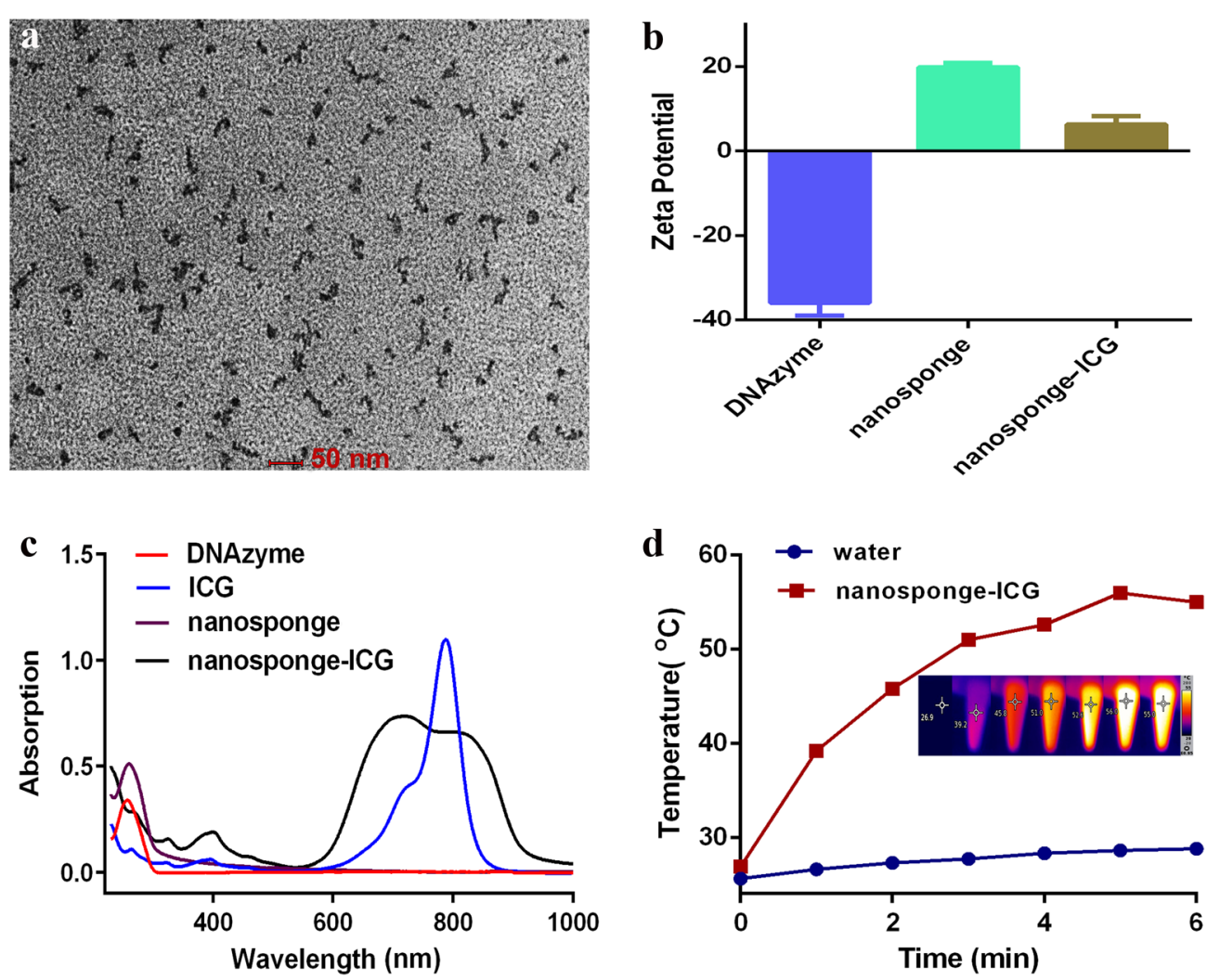

Fig. 1 Characterization of nanosponge-ICG. a TEM images of as-prepared nanosponge-ICG; b zeta potential of nanosponge-ICG; c UV-Vis absorbance spectra of free DNAzyme, ICG, nanosponge, and nanosponge-ICG; $\mathbf{d}$ temperature change curves and IR thermal imaging of nanospongeICG upon $808 \mathrm{~nm}$ light irradiation for $6 \mathrm{~min}$ at a power intensity of $0.4 \mathrm{~W} / \mathrm{cm}^{2}$

photothermal heating effect increased monotonically with these two parameters. These results indicate that the nanosponge-ICG possesses good photothermal properties.

\section{Internalization of DNAzyme-nanosponge}

The nanosponge-ICG was stable with a zeta potential of $+6 \mathrm{mV}$. In addition, the nanosponge-ICG could effectively protect DNA from degradation and promote efficient cellular uptake. HSP70 is a survival protein that is overexpressed in various malignant tumors, and its expression correlates with increased cell proliferation, poor differentiation, and poor therapeutic outcomes in human breast cancer ${ }^{29}$. In particular, the expression of HSP70 is upregulated substantially by heat stress in breast cancer cells to protect these cells from damage, resulting in the failure of thermal therapy ${ }^{17,18,25}$. Here, MCF-7 cells were chosen as model cells to investigate the PTT efficiency of DNAzyme-nanosponges. The nanosponge-ICG was labeled with FITC to facilitate the fluorescence observation of cellular uptake under CLSM. As shown in Fig. 2a, bright green and purple fluorescence (ICG) were present in the same MCF-7 cells, which demonstrated the efficient internalization of nanosponge-ICG by MCF-7 cells. Flow cytometry tests further demonstrated their efficient cellular uptake (Fig. 2b). Because the main gene silencing location was in the cytoplasm, we monitored the track of the nanosponges at different time points. Previous studies have indicated that many therapeutic nanoparticles enter cells via the endosomal pathway and localize in the lysosome ${ }^{30}$. However, the lysosomal escape of these nanoparticles remained an issue that affected their therapeutic efficiency. The results in Fig. 2c show that the green color of the nanosponges and the red color of the lysosome are co-located at the same position at $2 \mathrm{~h}$. Then, these two colors separated over time, indicating the successful escape of these nanoparticles from the lysosome. This result was due to the protonation of the PEI in the nanosponge, which promoted the escape of the nanoparticles from the lysosome ${ }^{31}$. After demonstrating the internalization pathway of the nanosponges, we investigated the impact of the acidic lysosomal environment on the catalytic capacity of the nanosponges. As the DNAzymes were encoded in long single-stranded DNA, the release of these DNAzymes was different from that of short oligonucleotides, such as siRNA. The cationic polymer PEI in the nanosponge became protonated, 

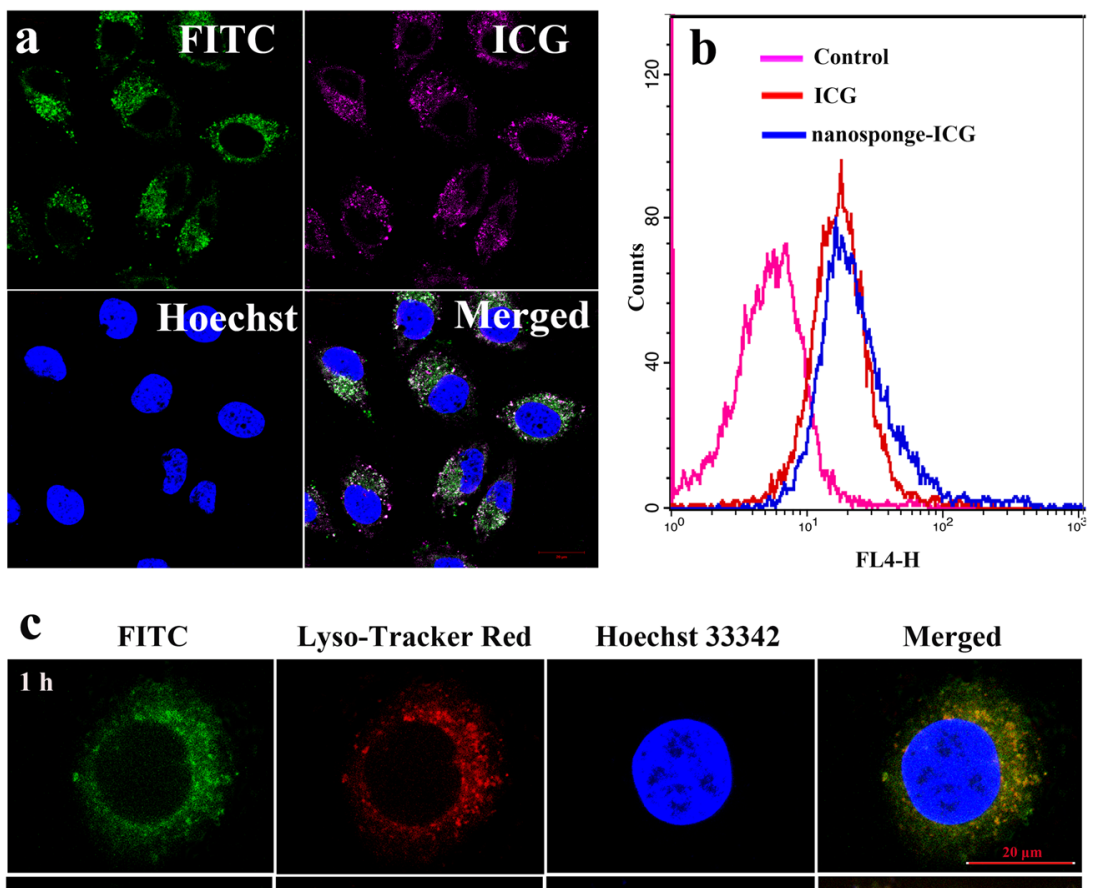

Lyso-Tracker Red

Hoechst 33342
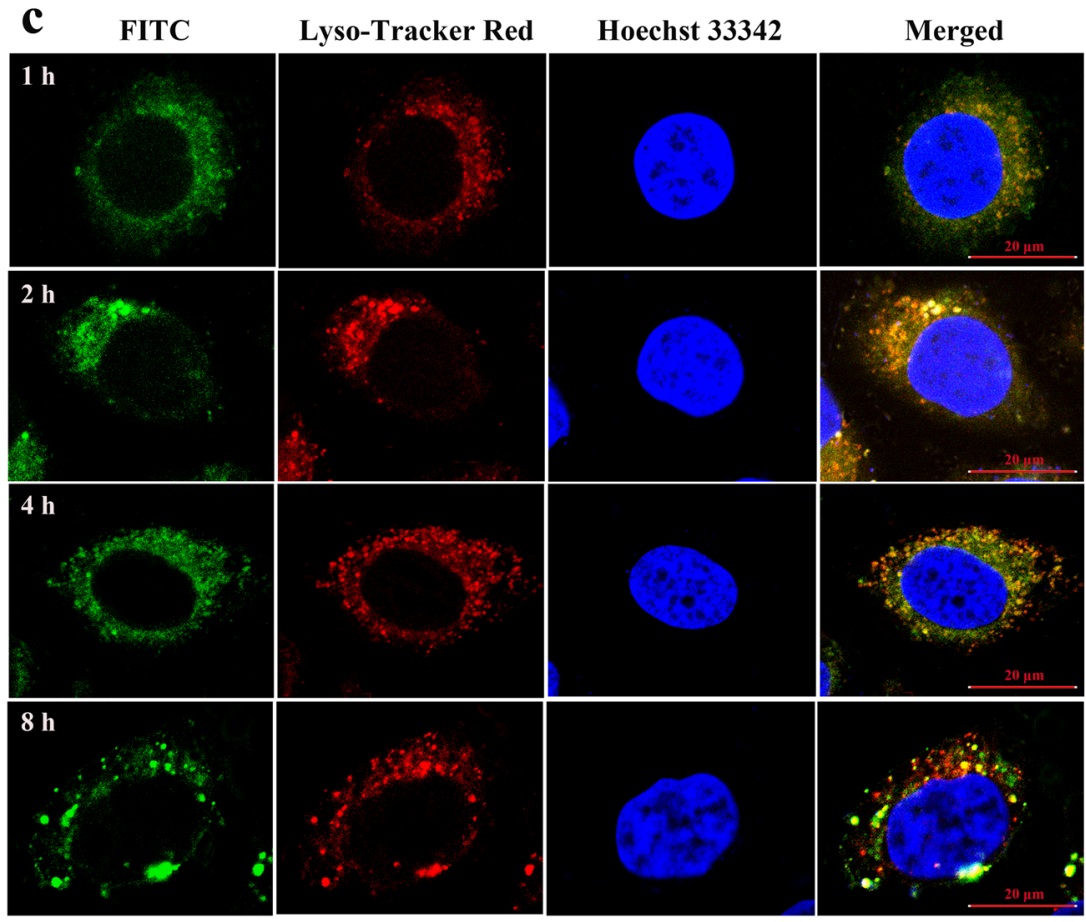

Fig. 2 The uptake of DNAzyme-nanosponge. a CLSM images showing cellular uptake of nanosponge-ICG; $\mathbf{b}$ flow cytometry histogram profile of MCF-7 cells incubated with ICG or nanosponge-ICG; c CLSM images of MCF-7 cells treated with nanosponge-ICG at different time points.Green: FITClabeled DNAzyme; red: Lyso-Tracker Red-stained lysosome; blue: Hoechst 33342-stained nucleus. Bar: 20 MM

swelled, and dissociated from the long single-stranded DNA when they were incubated in acidic buffer. Once the embedded DNAzyme sequences were able to contact their reaction substrates, their catalytic capacity recovered. As shown in Fig. S5, the catalytic capacity recovered with increasing incubation time in the acidic lysosomal environment.

\section{Inhibition of the proliferation of cancer cells by DNAzyme- nanosponge}

HSP70 exists in nearly all living organisms and is upregulated by heat stress to protect cells from thermal damage $^{32}$. As shown in Fig. S6, the expression of HSP70 increased significantly $1 \mathrm{~h}$ after irradiation and was nearly 3.2 times that of the control cells at $4 \mathrm{~h}$. These results demonstrate that a heat shock response occurred in MCF7 cells under ICG-mediated PTT, protecting the cells from hyperthermic damage and potentially impacting the PTT efficacy. To confirm the effectiveness of using nanosponge-ICG as a photothermal therapeutic agent, the 3-(4,5-dimethylthiazol-2-yl)-2,5-diphenyltetrazolium bromide (MTT) assay against the MCF-7 cell line under NIR light irradiation was carried out. The results shown in Fig. 3a indicate that no significant cytotoxicity was 


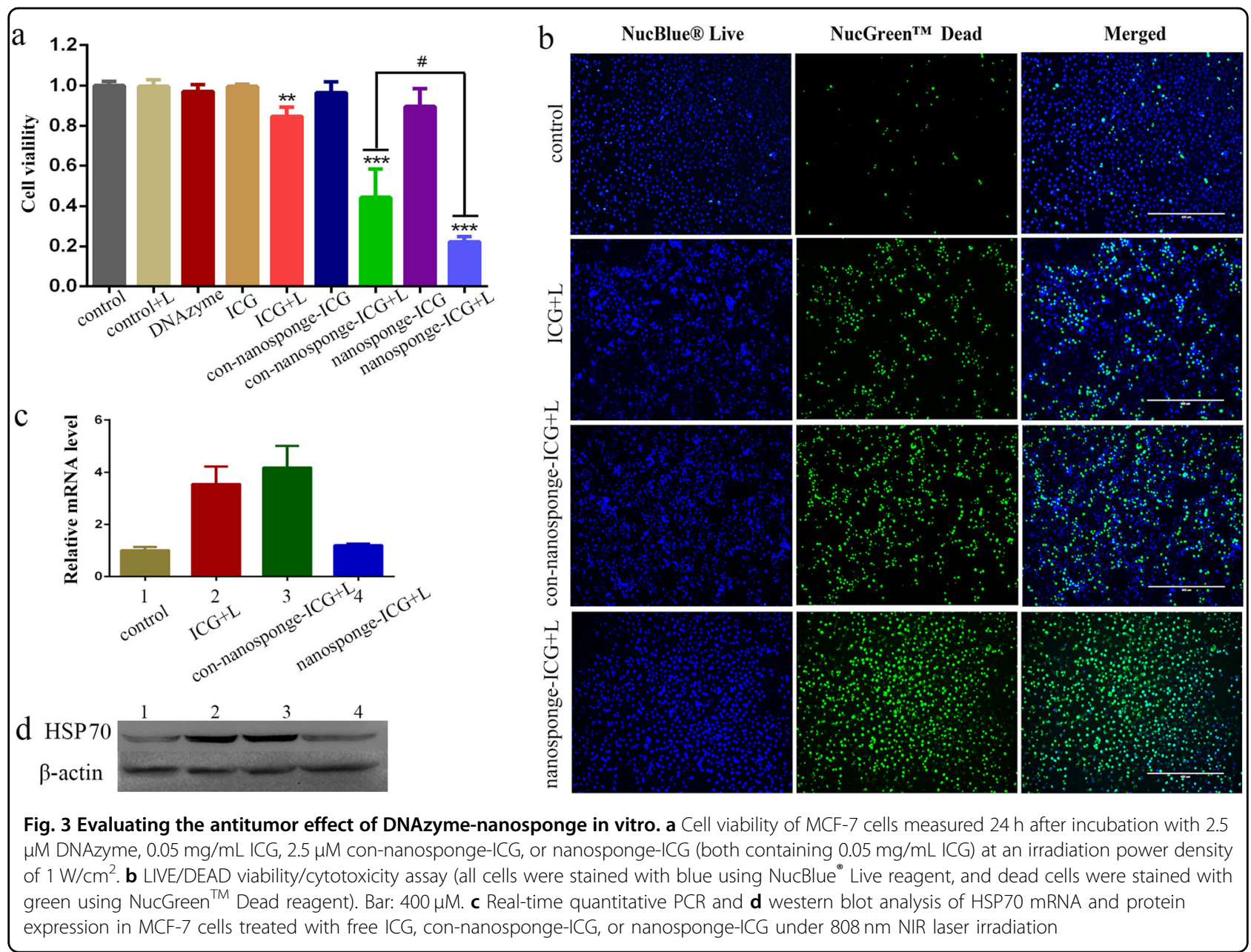

observed upon incubation with DNAzyme, ICG, or nanosponge-ICG. It is important to note that the photothermal conversion efficiency of ICG decreases over time due to its photodegradability ${ }^{33}$. As expected, only $20 \%$ of the MCF-7 cells treated with ICG were killed under NIR irradiation at a power density of $1 \mathrm{~W} / \mathrm{cm}^{2}$. However, the viability of the cells treated with con-nanosponge-ICG decreased substantially upon NIR light irradiation, indicating the efficient intracellular ICG delivery and protection of these agents. In comparison to the previously mentioned experiments without HSP70 mRNA interference, the viability was further decreased from 43 to $22 \%$ by incubation with nanosponge-ICG, indicating that DNAzyme-based HSP70 gene silencing can increase the sensitivity of MCF-7 cells to heat. After irradiation and 24 $h$ incubation, the cells were stained using a LIVE/DEAD fixable dead cell stain kit (Life Technologies, Grand Island, NY, USA). The living and dead cells were stained blue and green, respectively. The images in Fig. 3b indicate that more cells were killed by incubation with nanosponge-ICG under NIR light irradiation than in the
nanosponge-ICG or ICG group, which is consistent with the MTT results. Next, the HSP70 mRNA silencing efficiency of nanosponge-ICG in PTT was investigated using real-time quantitative PCR and western blots (Fig. 3c, d). Under $808 \mathrm{~nm}$ laser irradiation, HSP70 was substantially overexpressed at both the mRNA and protein levels in the ICG and con-nanosponge-ICG groups. After silencing of the HSP70 gene using the DNAzymes embedded in the nanosponge, the expression decreased to a normal level. Therefore, the multivalent DNAzymes may act as an efficient gene silencing agent to sensitize MCF-7 cells to heat, improving the PTT efficiency.

\section{In vivo location of DNAzyme-nanosponge}

After demonstrating their high HSP70 gene silencing capability and enhanced photothermal therapeutic efficiency, we used these nanosponges for in vivo cancer therapy. The enrichment of nanosponge-ICG in tumor tissue is a prerequisite for successful gene silencing in vivo, and therefore the distribution of nanosponge-ICG in mice was examined. MCF-7 xenograft BALB/c node 

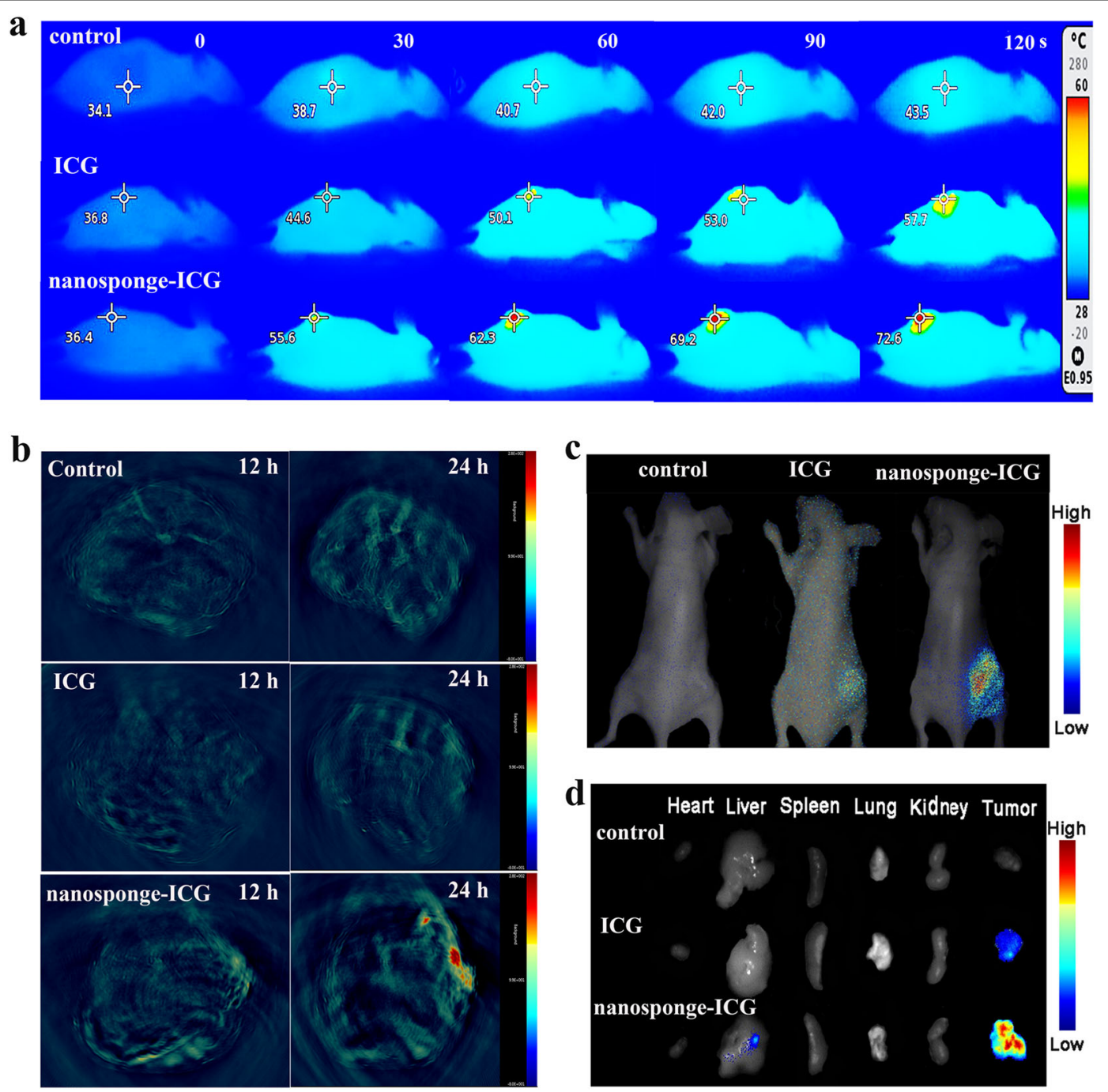

Fig. 4 Biodistribution of DNAzyme-nanosponge in tumor-bearing mice. a In vivo photothermal images of DNAzyme, ICG, con-nanosponge-ICGtreated, and nanosponge-ICG-treated mice exposed to $808 \mathrm{~nm}$ laser with a power density of $2 \mathrm{~W} / \mathrm{cm}^{2} \cdot \mathbf{b}$-d Biodistribution of ICG and nanospongeICG. b PA imaging of tumors from saline-treated, $0.1 \mathrm{mg} / \mathrm{mL}$ ICG-treated, and $5 \mu \mathrm{M}$ nanosponge-treated mice 12 and $24 \mathrm{~h}$ after intravenous injection. c Fluorescence imaging of mice acquired $24 \mathrm{~h}$ after intravenous injection. $\mathbf{d}$ Fluorescence imaging of main organs treated with ICG and nanospongeICG $24 \mathrm{~h}$ after intravenous injection

mice with a maximum tumor volume of $100 \mathrm{~mm}^{3}$ were intravenously injected with DNAzyme, ICG, con-nanosponge-ICG, or nanosponge-ICG. After $24 \mathrm{~h}$, the tumors in the mice were irradiated with an $808 \mathrm{~nm}$ laser at a power density of $2 \mathrm{~W} / \mathrm{cm}^{2}$ for $10 \mathrm{~min}$. Due to the loading of ICG and deep penetration of the NIR light, the nanosponges have the potential for photothermal imaging and photoacoustic (PA) imaging to monitor the accumulation of nanosponge-ICG in tumor tissues. As shown in Fig. 4a, an increase in temperature was observed in the nanospongeICG group. However, the increase in temperature was much lower in the ICG group. Similar results were obtained using PA imaging. The results in Fig. 4b indicate that a strong PA signal appeared at the tumor site at $24 \mathrm{~h}$ in the nanosponge-ICG group. In contrast, a much lower PA signal was observed in the ICG group. Nanoparticles that range from 20 to $200 \mathrm{~nm}$ may accumulate in tumors via the EPR effect, but small molecules tend to be rapidly cleaned up ${ }^{34}$. This phenomenon contributed to the remarkable difference between the ICG and nanospongeICG groups. The as-developed nanosponges could also be used for fluorescent imaging to determine their distribution in the major organs. The results in Fig. 4c indicate that fluorescence appeared in the tumor section in the nanosponge-ICG group, which was consistent with the photothermal imaging and PA imaging results. In addition, the major organs of mice injected with ICG or nanosponge-ICG were harvested $24 \mathrm{~h}$ after injection for 
a

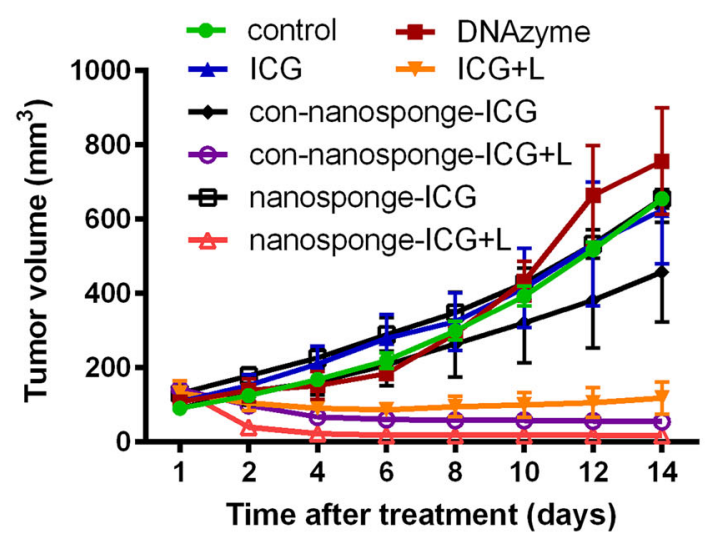

b

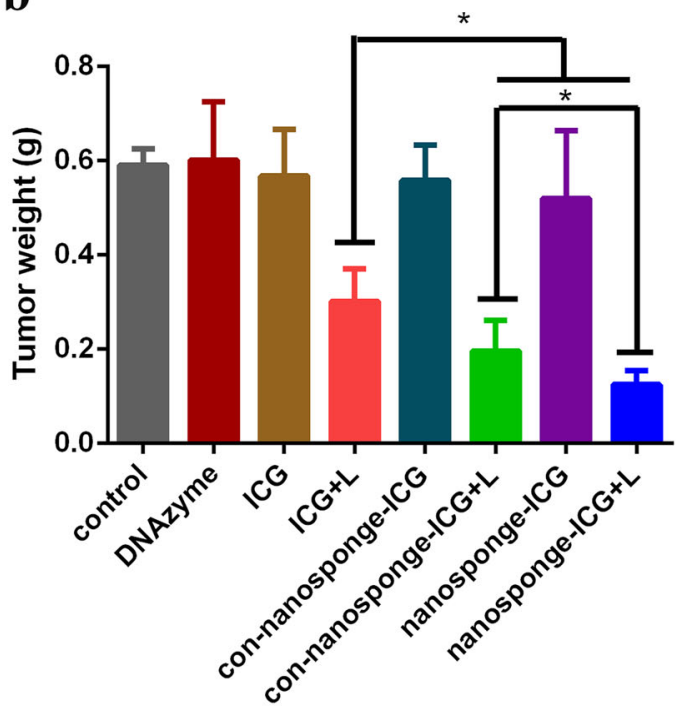

c
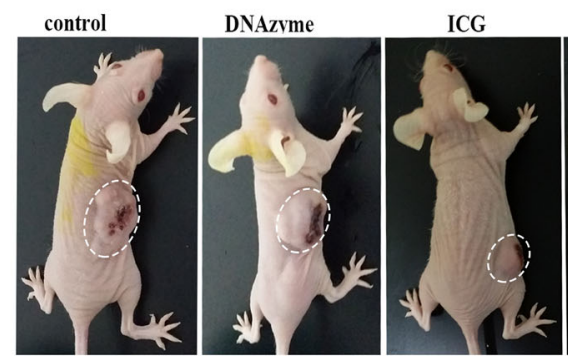

ICG+L
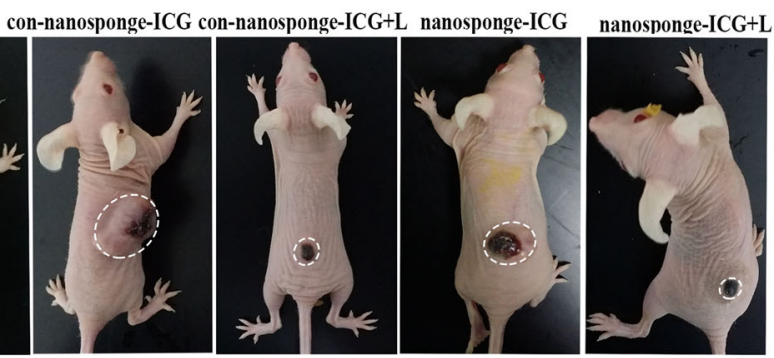

d

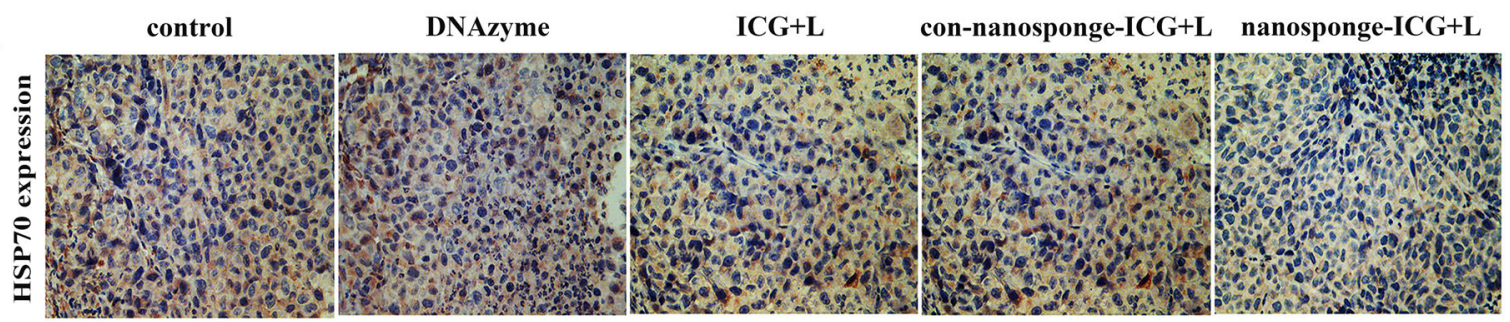

e

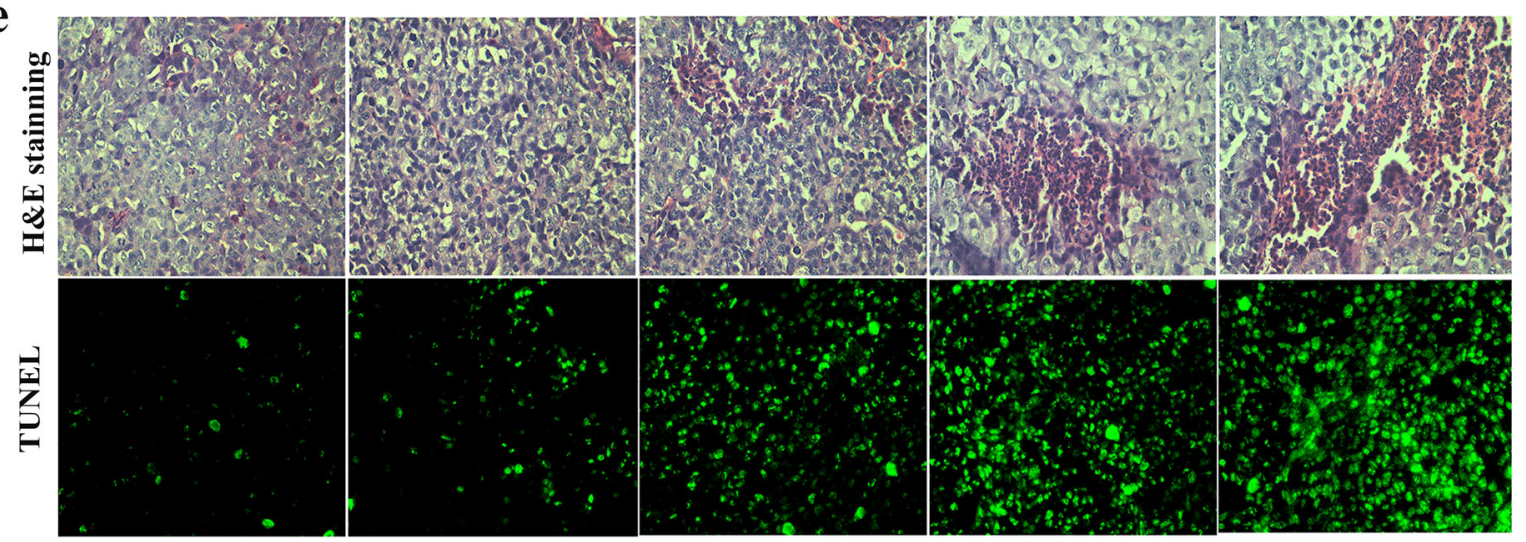

Fig. 5 Nanosponge-ICG improved PTT by overcoming thermoresistance. a Tumor growth curves of xenograft model mice. $\mathbf{b}$ Average tumor weight of different groups measured 15 days after treatment. $\mathbf{c}$ Images of tumor-bearing mice after different treatments at 14 days. $\mathbf{d}$ Expression of HSP70 in tumors from different groups $24 \mathrm{~h}$ after treatment, as determined by immunochemistry (400X). e Representative images of H\&E-stained tumor sections and apoptotic cells of tumors from different groups, as determined by TUNEL assay (400x) 
ex vivo imaging. As shown in Fig. 4d and S7, the fluorescence in the tumor tissues was stronger than that in other organs in both the ICG and nanosponge-ICG groups $24 \mathrm{~h}$ after intravenous administration. These results suggest that nanosponge-ICG, with its high tumor accumulation, is a promising theranostic agent for simultaneous thermal, PA, and fluorescence imaging.

\section{In vivo PTT}

To confirm the in vivo gene silencing and PTT efficacy of nanosponge-ICG, female xenograft nude mice were randomly divided into eight groups and intravenously injected with different samples. At $24 \mathrm{~h}$ after injection, the tumors of the mice treated with ICG, con-nanospongeICG, and nanosponge-ICG were irradiated with an 808 $\mathrm{nm}$ laser at a power density of $2 \mathrm{~W} / \mathrm{cm}^{2}$ for $10 \mathrm{~min}$. After $24 \mathrm{~h}$, the tumors were harvested, and the expression of HSP70 was measured by immunohistochemistry. As shown in Fig. 5d and Table S3, the HSP70 protein expression increased significantly in the ICG and connanosponge-ICG groups after NIR light irradiation, suggesting the occurrence of thermal resistance in the tumors. In contrast, no obvious elevated expression of the HSP70 protein was observed in the nanosponge-ICG group. The lower expression of HSP70 in the nanosponge-ICG group may be due to the targeted silencing effects of the HSP70 DNAzyme delivered by the nanosponge to the tumor. The tumor volume measured for each group was also plotted as a function of time in Fig. 5a. The mice treated with DNAzyme, ICG, connanosponge-ICG, or nanosponge-ICG exhibited rapid tumor growth that was similar to the trend observed for the control group. This result suggested that free DNAzyme, ICG, and nanosponge-ICG were not toxic to the cancer cells. In ICG $+\mathrm{L}$ or con-nanosponge-ICG $+\mathrm{L}$ group, tumor growth was decreased due to the photothermal heat. However, the thermal resistance of the tumor cells increased due to the unsatisfactory photothermal therapeutic effect, resulting in the recurrence of the tumor and eventual failure of the treatment. In contrast, by silencing the HSP70 gene using multivalent DNAzymes in the nanosponge, the photothermal therapeutic efficiency was improved (Fig. 5a, b, c). Due to the inhibited expression of the HSP70 protein, the sensitivity of the cancer cells to heat increased, which improved the photothermal therapeutic efficiency. Next, H\&E staining was used to assess the pathological morphology of the tumor tissues. Distinctive features of cellular damage including abundant pyknosis, coagulative necrosis, and considerable regions of karyolysis were observed in the laser-treated tumor tissue obtained from mice treated with the nanosponge-ICG group. However, the damage was slight in tumors from the ICG $+\mathrm{L}$ or con-nanosponge-ICG $+\mathrm{L}$ group (Fig. 5e). Then, terminal deoxynucleotidyl transferase-mediated dUTP nick-end labeling (TUNEL) was used to determine the levels of necrosis and apoptosis. More green fluorescent cells were observed in the ICG $+\mathrm{L}$, con-nanosponge-ICG $+\mathrm{L}$, and nanosponge-ICG $+\mathrm{L}$ groups than in the control group, implying that ICG-mediated PTT could cause cell death via activating apoptosis. In the nanosponge-ICG $+\mathrm{L}$ group, the number of green fluorescent cells increases substantially, indicating that more cells undergo apoptosis at the same laser power. Therefore, the increased sensitivity of cancer cells toward photothermal heat was due to nanosponge-ICG-mediated HSP70 silencing, which resulted in additional cancer cell apoptosis. As a control experiment, mice with bilateral tumors (on the left and right flanks) were intratumorally injected with ICG (left) and nanosponge-ICG (right) to investigate the photothermal effects. Solutions consisting of $100 \mu \mathrm{L}$ of ICG and nanosponge-ICG containing $0.1 \mathrm{mg} / \mathrm{mL}$ ICG were injected into the center of each tumor. The surface temperature of the right tumor, which was injected with nanosponge-ICG, increased rapidly within $120 \mathrm{~s}$ under laser irradiation administered $8 \mathrm{~h}$ post injection (Fig. S8). Due to the long retention effect of nanosponge-ICG and the downregulation of the HSP70 protein, the right group exhibited an antitumor effect (Fig. S9). Therefore, nanosponge-ICG acts as an efficient therapeutic agent to overcome thermal resistance by DNAzyme-based HSP70 gene silencing.

\section{Biosafety analysis of DNAzyme-nanosponge}

Potential in vivo toxicity is one primary concern that needs to be investigated prior to the practical application of nanomaterials. The biocompatibility of nanospongeICG with blood cells was determined by testing the hemolytic behaviors of these nanomaterials at different concentrations. The results shown in Fig. S10 indicate that almost no hemolysis of blood cells was detected even at a concentration as high as $10 \mu \mathrm{M}$. In addition, serum was collected from each group to evaluate the in vivo toxicity 15 days after injection. Eight important cardiac, hepatic, and kidney function indicators (ALT, AST, UA, BUN, Cr, LDH, HBDH, CK) were measured. All the indicators shown in Table S4 were in the reference ranges and exhibited no significant differences between the nanosponge-ICG and control groups, which confirmed that the nanosponge-ICG therapeutic agent exhibited good biocompatibility in this test. Histological analysis of the heart, liver, spleen, lung, and kidney indicated no abnormalities in the tissue structures, revealing that no tissue damage, lesions, or inflammation was observed after nanosponge-ICG injection (Fig. S11). Therefore, these results demonstrate the non-toxicity and biocompatibility of these nanotherapeutic agents as well as their promise for use in clinical applications. 


\section{Conclusion}

In summary, we have developed a nanosponge-ICG therapeutic platform for highly efficient PTT using DNAzymes to overcome thermal resistance. The nanosponge was constructed by simply assembling a cationic polymer and a long single strand of DNA, which can be customized with multivalent DNAzyme sequences using the RCA method. The multivalent DNAzyme can efficiently silence the HSP70 gene to sensitize MCF-7 cells to heat, eliminating the heat shock response of cancer cells. In vitro studies demonstrated that this nanosponge-ICG therapeutic platform can downregulate the expression of HSP70 genes or proteins to normal levels upon photothermal treatment and thereby enhance the therapeutic efficiency. In addition, the nanosponge-ICG can act as an efficient multimode imaging agent to trace its accumulation in tumor tissue. The in vivo antitumor results indicated that tumor growth can be restrained by PTT without adverse side effects after the injection of nanosponge-ICG, indicating the in vivo effectiveness of the nanosponge as a gene silencing agent to sensitize MCF-7 cells to heat. Due to its biocompatibility and high efficiency, nanosponge-ICG is a promising nanotherapeutic agent for PTT.

\section{Acknowledgements \\ The work was supported by the National Natural Science Foundation of China (21271059, 31470961, 21601046, 31500812, and 21603051), the Science and Technology Research Project of Higher Education Institutions in Hebei Province (QN2015132), the Foundation for Returned Overseas Chinese Scholars of Hebei Province (CY201703), and the Natural Science Foundation of Hebei Province (B2015201097, B2016201169, B2016201031, B2017201226, B2018201221, and B2018201157).}

\section{Conflict of interest}

The authors declare that they have no conflict of interest.

\section{Publisher's note}

Springer Nature remains neutral with regard to jurisdictional claims in published maps and institutional affiliations.

Supplementary information is available for this paper at https://doi.org/ 10.1038/s41427-018-0040-7.

Received: 9 November 2017 Revised: 10 February 2018 Accepted: 19 March 2018.

Published online: 1 May 2018

\section{References}

1. Holohan, C., Van Schaeybroeck, S., Longley, D. B. \& Johnston, P. G. Cancer drug resistance: an evolving paradigm. Nat. Rev. Cancer 13, 714-726 (2013).

2. Ramos, P. \& Bentires-Alj, M. Mechanism-based cancer therapy: resistance to therapy, therapy for resistance. Oncogene 34, 3617-3626 (2015).

3. Wang, Z., Xu, Z. \& Zhu, G. A platinum(IV) anticancer prodrug targeting nucleotide excision repair to overcome cisplatin resistance. Angew. Chem. Int. Ed. 55, 15564-15568 (2016).

4. Fan, $\mathrm{H}$. et al. A smart DNAzyme- $\mathrm{MnO}_{2}$ nanosystem for efficient gene silencing. Angew. Chem. Int. Ed. 54, 4801-4805 (2015).

5. Zhao, Y. et al. PolyMetformin combines carrier and anticancer activities for in vivo siRNA delivery. Nat. Commun. 7, 11822 (2016).
6. Parak, W. J. Nanomaterials. Controlled interaction of nanoparticles with cells. Science 351, 814-815 (2016).

7. Song, J. et al. Gold nanoparticle coated carbon nanotube ring with enhanced raman scattering and photothermal conversion property for theranostic applications. J. Am. Chem. Soc. 138, 7005-7015 (2016).

8. Cheng, L., Wang, C., Feng, L., Yang, K. \& Liu, Z. Functional nanomaterials for phototherapies of cancer. Chem. Rev. 114, 10869-10939 (2014).

9. Shanmugam, V., Selvakumar, S. \& Yeh, C. S. Near-infrared light-responsive nanomaterials in cancer therapeutics. Chem. Soc. Rev. 43, 6254-6287 (2014).

10. Huang, P. et al. Biodegradable gold nanovesicles with an ultrastrong plasmonic coupling effect for photoacoustic imaging and photothermal therapy. Angew. Chem. Int. Ed. 52, 13958-13964 (2013).

11. Song, J. et al. Ultrasmall gold nanorod vesicles with enhanced tumor accumulation and fast excretion from the body for cancer therapy. Adv. Mater. 27, 4910-4917 (2015).

12. Robinson, J. T. et al. Ultrasmall reduced graphene oxide with high nearinfrared absorbance for photothermal therapy. J. Am. Chem. Soc. 133, 6825-6831 (2011).

13. Dong, K., Liu, Z., Li, Z., Ren, J. \& Qu, X. Hydrophobic anticancer drug delivery by a $980 \mathrm{~nm}$ laser-driven photothermal vehicle for efficient synergistic therapy of cancer cells in vivo. Adv. Mater. 25, 4452-4458 (2013).

14. Wu, Z., Li, W., Luo, C., Su, C. \& Yeh, C. Rattle-type Fe $\mathrm{O}_{4} @ C u S$ developed to conduct magnetically guided photoinduced hyperthermia at first and second NIR biological windows. Adv. Funct. Mater. 25, 6430-6430 (2015).

15. Yang, $X$. et al. Near-infrared light-triggered, targeted drug delivery to cancer cells by aptamer gated nanovehicles. Adv. Mater. 24, 2890-2895 (2012).

16. Kolemen, S. et al. Remote-controlled release of singlet oxygen by the plasmonic heating of endoperoxide-modified gold nanorods: towards a paradigm change in photodynamic therapy. Angew. Chem. Int. Ed. 55, 3606-3610 (2016).

17. Wang, S. et al. Selectively sensitizing malignant cells to photothermal therapy using a CD44-targeting heat shock protein 72 depletion nanosystem. ACS Nano 10, 8578-8590 (2016).

18. Yoo, D., Jeong, H., Noh, S. H., Lee, J. H. \& Cheon, J. Magnetically triggered dual functional nanoparticles for resistance-free apoptotic hyperthermia. Angew. Chem. Int. Ed. 52, 13047-13051 (2013).

19. Wang, B. K. et al. Gold-nanorods-siRNA nanoplex for improved photothermal therapy by gene silencing. Biomaterials 78, 27-39 (2016).

20. Yang, L. et al. Photothermal therapeutic response of cancer cells to aptamer gold nanoparticle-hybridized graphene oxide under NIR illumination. ACS Appl. Mater. Interfaces 7, 5097-5106 (2015).

21. Khoei, S., Goliaei, B., Neshasteh-Riz, A. \& Deizadji, A. The role of heat shock protein 70 in the thermoresistance of prostate cancer cell line spheroids. FEBS Lett. 561, 144-148 (2004).

22. Bakthisaran, R., Tangirala, R. \& Rao, C. M. Small heat shock proteins: role in cellular functions and pathology. Biochim. Biophys. Acta 1854, 291-319 (2015).

23. Liu, D. et al. Nanogel-encapsulated PEDOT and HSP70 inhibitor for improving the depth of the photothermal therapeutic effect. Adv. Funct. Mater. 26, 4749-4759 (2016)

24. Wang, L. et al. Cypate-conjugated porous upconversion nanocomposites for programmed delivery of heat shock protein 70 small interfering RNA for gene silencing and photothermal ablation. Adv. Funct. Mater. 26, 3480-3489 (2016).

25. Ali, M. R., Ali, H. R., Rankin, C. R. \& El-Sayed, M. A. Targeting heat shock protein 70 using gold nanorods enhances cancer cell apoptosis in low dose plasmonic photothermal therapy. Biomaterials 102, 1-8 (2016).

26. Jin, $Y$. et al. Biodegradable, multifunctional DNAzyme nanoflowers for enhanced cancer therapy. NPG Asia Mater. 9, e365 (2017).

27. Zhao, P. et al. Improving drug accumulation and photothermal efficacy in tumor depending on size of ICG loaded lipid-polymer nanoparticles. Biomaterials 35, 6037-6046 (2014).

28. Zhao, W., Ali, M. M., Brook, M. A. \& Li, Y. Rolling circle amplification: applications in nanotechnology and biodetection with functional nucleic acids. Angew. Chem. Int. Ed. 47, 6330-6337 (2008).

29. Barnes, J. A., Dix, D. J., Collins, B., Luft, W. C. \& Allen, J. W. Expression of inducible Hsp70 enhances the proliferation of MCF-7 breast cancer cells and protects against the cytotoxic effects of hyperthermia. Cell Stress Chaperons 6, 316 (2001).

30. Wang, F. et al. Efficient RNA delivery by integrin-targeted glutathione responsive polyethyleneimine capped gold nanorods. Acta Biomater. 23, 136-146 (2015). 
31. Akinc, A., Thomas, M., Klibanov, A. M. \& Langer, R. Exploring polyethyleniminemediated DNA transfection and the proton sponge hypothesis. J. Gene. Med. 7, 657-663 (2005).

32. Ciocca, D. R., Fanelli, M. A., Cuello-Carrion, F. D. \& Castro, G. N. Heat shock proteins in prostate cancer: from tumorigenesis to the clinic. Int. J. Hyperth. 26, 737-747 (2010).
33. Kuo, W. S. et al. Gold nanomaterials conjugated with indocyanine green for dual-modality photodynamic and photothermal therapy. Biomaterials $\mathbf{3 3}$ 3270-3278 (2012).

34. Albanese, A., Tang, P. S. \& Chan, W. C. W. The effect of nanoparticle size, shape, and surface chemistry on biological systems. Annu. Rev. Biomed. Eng. 14, 1-16 (2012). 